\title{
Gravitino thermal production revisited
}

\author{
Helmut Eberl®, ${ }^{1, *}$ Ioannis D. Gialamas $\oplus^{2, \dagger}$ and Vassilis C. Spanos $\oplus^{2, \ddagger}$ \\ ${ }^{1}$ Institut für Hochenergiephysik der Österreichischen Akademie der Wissenschaften, \\ A-1050 Vienna, Austria \\ ${ }^{2}$ National and Kapodistrian University of Athens, Department of Physics, \\ Section of Nuclear \& Particle Physics, GR-157 84 Athens, Greece
}

(Received 4 November 2020; revised 1 February 2021; accepted 22 March 2021; published 23 April 2021)

\begin{abstract}
We calculate the gravitino production rate, computing its one-loop thermal self-energy. Gravitino production processes that do not result through thermal cuts of its self-energy, have been identified and taken into account. Correcting analytical errors and numerical approximations in the previous calculations, we present our result. This deviates from the latest estimation by almost $10 \%$. More importantly, we provide a convenient formula, for calculating the gravitino production rate and its thermal abundance, as a function of the reheating temperature of the Universe.
\end{abstract}

DOI: 10.1103/PhysRevD.103.075025

\section{INTRODUCTION}

Extensions of the Standard Model (SM) in the context of supergravity provide us with a dark matter (DM) candidate particle, the gravitino, the superpartner of graviton. It interacts purely gravitationally with other particles and thus naturally escapes direct or indirect detection, as the current experimental and observational data on DM searches suggest. Therefore, the precise knowledge of its cosmological abundance is essential to apply cosmological constraints on these models. Gravitinos may be produced in various ways: (i) nonthermally, from the inflaton decays [1-8], (ii) much later around the big bang nucleosynthesis epoch, through the decays of unstable particles [9-12], and (iii) last but not least, thermally, using a freeze-in production mechanism, as the Universe cools down from the reheating temperature $\left(T_{\text {reh }}\right)$ until now [6,13-27]. In particular, assuming gauge mediated supersymmetry breaking, a different production mechanism (freeze-out) has to be employed [28-31].

The effort in calculating the thermal gravitino abundance, using various techniques, methods, and approximations, spans over almost the last four decades. Since the gravitinos are mainly thermally produced at very high temperatures, the effective theory of light gravitinos, the socalled nonderivative approach, involving only the spin $1 / 2$

\footnotetext{
*helmut.eberl@oeaw.ac.at

i.gialamas@phys.uoa.gr

*vspanos@phys.uoa.gr
}

Published by the American Physical Society under the terms of the Creative Commons Attribution 4.0 International license. Further distribution of this work must maintain attribution to the author(s) and the published article's title, journal citation, and DOI. Funded by SCOAP.
Goldstino components, was initially used. In this context, as some of the production amplitudes exhibit infrared (IR) divergences, they were regularized by introducing either a finite thermal gluon mass or an angular cutoff. Thus, in [14], the basic $2 \rightarrow 2$ gravitino production processes had been tabulated for the first time and calculated; see Table I. This calculation was further improved in [16,17].

As the Braaten, Pisarski, Yuan (BPY) method [32,33] succeeded in calculating the axion thermal abundance, in [19] it was further applied to the gravitino, motivated by the fact that the gravitinolike axion interacts extremely weakly with the rest of the spectrum. Although in [20] the previous IR regularization technique was used, in [21,24] the BPY method was employed, taking in addition into account the contribution of the spin $3 / 2$ pure gravitino components.

Eventually, in [26], the calculation method improved significantly. There it was argued that the basic requirement to apply the BPY prescription, i.e., $g \ll 1$, where $g$ is the gauge coupling constant, is not satisfied in the whole temperature range of the calculation, especially if $g$ is the strong coupling constant $g_{3}$. Therefore, the authors calculated the one-loop thermal gravitino self-energy numerically beyond the hard thermal loop approximation, with the benefit that this incorporates also the $1 \rightarrow 2$ processes besides the $2 \rightarrow 2$ ones. More importantly, it was noticed that the so-called subtracted part, i.e., pieces of the $2 \rightarrow 2$ squared amplitudes for which the self-energy may not account for, are IR finite. The main numerical result in [26] on the gravitino production rate differs significantly, almost by a factor of 2 , with respect to the previous works [24,27]. Unfortunately, in [26], the main analytical results appear to be inadequate. In particular, in Sec. IV A, the equations on the self-energy contribution for the gravitino production rate seem to be inconsistent even dimensionally. In addition, the 
TABLE I. Squared matrix elements for gravitino production in $S U(3)_{c}$ in terms of $g_{3}^{2} Y_{3} / M_{\mathrm{P}}{ }^{2}$ assuming massless particles, $Y_{3}=1+m_{\tilde{g}}^{2} /\left(3 m_{3 / 2}^{2}\right), C_{3}=24$, and $C_{3}^{\prime}=48$.

\begin{tabular}{lccc}
\hline \hline $\mathrm{X}$ & Process & $\left|\mathcal{M}_{X, \text { full }}\right|^{2}$ & $\left|\mathcal{M}_{X, \text { sub }}\right|^{2}$ \\
\hline $\mathrm{A}$ & $g g \rightarrow \tilde{g} \tilde{G}$ & $4 C_{3}\left(s+2 t+2 t^{2} / s\right)$ & $-2 s C_{3}$ \\
$\mathrm{~B}$ & $g \tilde{g} \rightarrow g \tilde{G}$ & $-4 C_{3}\left(t+2 s+2 s^{2} / t\right)$ & $2 t C_{3}$ \\
$\mathrm{C}$ & $\tilde{q} g \rightarrow q \tilde{G}$ & $2 s C_{3}^{\prime}$ & 0 \\
$\mathrm{D}$ & $g q \rightarrow \tilde{q} \tilde{G}$ & $-2 t C_{3}^{\prime}$ & 0 \\
$\mathrm{E}$ & $\tilde{q} q \rightarrow g \tilde{G}$ & $-2 t C_{3}^{\prime}$ & 0 \\
$\mathrm{~F}$ & $\tilde{g} \tilde{g} \rightarrow \tilde{g} \tilde{G}$ & $8 C_{3}\left(s^{2}+t^{2}+u^{2}\right)^{2} /(s t u)$ & 0 \\
$\mathrm{G}$ & $q \tilde{g} \rightarrow q \tilde{G}$ & $-4 C_{3}^{\prime}\left(s+s^{2} / t\right)$ & 0 \\
$\mathrm{H}$ & $\tilde{q} \tilde{g} \rightarrow \tilde{q} \tilde{G}$ & $-2 C_{3}^{\prime}\left(t+2 s+2 s^{2} / t\right)$ & 0 \\
$\mathrm{I}$ & $q \tilde{q} \rightarrow \tilde{g} \tilde{G}$ & $-4 C_{3}^{\prime}\left(t+t^{2} / s\right)$ & 0 \\
$\mathrm{~J}$ & $\tilde{q} \tilde{q} \rightarrow \tilde{g} \tilde{G}$ & $2 C_{3}^{\prime}\left(s+2 t+2 t^{2} / s\right)$ & 0 \\
\hline \hline
\end{tabular}

numerical estimation of this self-energy was computed only inside the light cone due to the limited computation resources of that time. Furthermore, two out of the four nonzero subtracted parts in the corresponding Table I in [26] turn out to be zero.

Motivated by these, in this paper, we recalculate the thermally corrected gravitino self-energy and we compute it without numerical approximations at the one-loop level. Eventually, since our final result for the gravitino production rate, like in [26], is numerical, following [6] we present an updated handy parametrization of this. Our final result differs from that shown in [26] approximately by $10 \%$. Moreover, we calculate the gravitino thermal abundance and discuss possible phenomenological consequences.

\section{THE SETUP}

As the gravitino is the superpartner of the graviton, its interactions are suppressed by the inverse of the reduced Planck mass $M_{\mathrm{P}}=(8 \pi G)^{-1 / 2}$. Hence, the dominant contributions to its production, in leading order of the gauge group couplings, are processes of the form $a b \rightarrow c \tilde{G}$, where $\tilde{G}$ stands for gravitino and $a, b, c$ can be three superpartners or one superpartner and two SM particles. The possible processes and the corresponding squared amplitudes in $S U(3)_{c}$ are given in Table I, where for their denotation by the letters A-J we follow the "historical" notation of [14]. In $S U(3)_{c}$, the particles $a, b$, and $c$ could be gluons $g$, gluinos $\tilde{g}$, quarks $q$, or/and squarks $\tilde{q}$. Analogous processes happen in $S U(2)_{L}$ or $U(1)_{Y}$, where the gluino mass $m_{\tilde{g}} \equiv M_{3}$ becomes $M_{2}$ or $M_{1}$, respectively. In the factor $Y_{N} \equiv 1+m_{\lambda_{N}}^{2} /\left(3 m_{3 / 2}^{2}\right)$, where $m_{\lambda_{N}}=$ $\left\{M_{1}, M_{2}, M_{3}\right\}$ and $m_{3 / 2}$ is the gravitino mass, the unity is related to the $3 / 2$ gravitino components and the rest to the $1 / 2$ Goldstino part. For the calculation of the spin $3 / 2$ part in the amplitudes, following [26], we have employed the gravitino polarization sum

$$
\Pi_{\mu \nu}^{3 / 2}(P)=\sum_{i= \pm 3 / 2} \Psi_{\mu}^{(i)} \bar{\Psi}_{\nu}^{(i)}=-\frac{1}{2} \gamma_{\mu} \not \gamma_{\nu}-\not P g_{\mu \nu}
$$

where $\Psi_{\mu}$ is the gravitino spinor and $P$ its momentum. As in [26], for the Goldstino spin $1 / 2$ part, the nonderivative approach is used $[18,27]$. The result for the full squared amplitude has been proved to be the same, either in the derivative or the nonderivative approach [34].

The Casimir operators in Table I are $C_{N}=\sum_{a, b, c}\left|f^{a b c}\right|^{2}=$ $N\left(N^{2}-1\right)=\{0,6,24\}$ and $C_{N}^{\prime}=\sum_{a, i, j}^{\phi}\left|T_{i j}^{a}\right|^{2}=\{11,21,48\}$, where $\sum_{a, i, j}^{\phi}$ denotes the sum over all involved chiral multiplets and group indices. $f^{a b c}$ and $T^{a}$ are the group structure constants and generators, respectively. Processes A, $\mathrm{B}$, and $\mathrm{F}$ are not present in $U(1)_{Y}$ because $C_{1}=0$. The masses for the particles $a, b$, and $c$ are assumed to be zero. In the third column of Table I, we present for each process the square of the full amplitude, which is the sum of individual amplitudes,

$$
\left|\mathcal{M}_{X, \text { full }}\right|^{2}=\left|\mathcal{M}_{X, s}+\mathcal{M}_{X, t}+\mathcal{M}_{X, u}+\mathcal{M}_{X, x}\right|^{2},
$$

where the indices $s, t, u$ indicate the diagrams which are generated by the exchange of a particle in the corresponding channel and the index $x$ stands for the diagram involving a quartic vertex. The so-called $D$-graph, following the terminology of [26], is illustrated in Fig. 1 for the case of the gluinogluon loop. Its contribution is the sum of the squared amplitudes for the $s, t$, and $u$ channel graphs,

$$
\left|\mathcal{M}_{X, D}\right|^{2}=\left|\mathcal{M}_{X, S}\right|^{2}+\left|\mathcal{M}_{X, t}\right|^{2}+\left|\mathcal{M}_{X, u}\right|^{2},
$$

plus $1 \rightarrow 2$ processes. This can be understood by applying the optical theorem. Hence, from the imaginary part of the loop graphs, one computes the sum of the decays $(1 \rightarrow 2)$ and the scattering amplitudes $(2 \rightarrow 2)$. In our case, we use resummed thermal propagators for the gauge boson and gaugino and by applying cutting rules one sees that $D$-graph describes both the scattering amplitudes appearing in (3) and decay amplitudes.

The subtracted part of the squared amplitudes is the difference between the full amplitudes (2) and the amplitudes already included in the $D$-graph (3), i.e.,

$$
\left|\mathcal{M}_{X, \text { sub }}\right|^{2}=\left|\mathcal{M}_{X, \text { full }}\right|^{2}-\left|\mathcal{M}_{X, D}\right|^{2} .
$$

For the processes $\mathrm{B}, \mathrm{F}, \mathrm{G}$, and $\mathrm{H}$, the corresponding amplitudes are IR divergent. For this reason, we follow the more elegant method comprising the separation of the total scattering rate into two parts, the subtracted and the $D$-graph part. It is worth to mention that for the processes with incoming or/and outgoing gauge bosons, we have checked explicitly the gauge invariance for $\left|\mathcal{M}_{X, \text { full }}\right|^{2}$. On the other hand, we note that $\left|\mathcal{M}_{X \text {,sub }}\right|^{2}$ is gauge dependent [35]. 
To sum up, the gravitino production rate $\gamma_{3 / 2}$ consists of three parts: (i) the subtracted rate $\gamma_{\text {sub }}$, (ii) the $D$-graph contribution $\gamma_{\mathrm{D}}$, and (iii) the top Yukawa rate $\gamma_{\text {top }}$,

$$
\gamma_{3 / 2}=\gamma_{\text {sub }}+\gamma_{\mathrm{D}}+\gamma_{\text {top }}
$$

Below, these three contributions are discussed in detail.

\section{A. The subtracted rate}

In the fourth column of Table I, we present the so-called subtracted part (4), which is the sum of the interference terms among the four types of diagrams $(s, t, u, x)$, plus the $x$-diagram squared, for each process. The subtracted part is nonzero only for the processes A and B. Note that in [26] the subtracted part for the processes $\mathrm{H}$ and $\mathrm{J}$ is also nonzero; we assume that the authors had used the squark-squark-gluino-Goldstino Feynman rule as given in [22], where a factor $\gamma_{5}$ is indeed missing. In contrast, we are using the correct Feynman rule as given in [27].

To calculate the subtracted rate for the processes $a b \rightarrow c \tilde{G}$, we use the general form

$$
\begin{aligned}
\gamma= & \frac{1}{(2 \pi)^{8}} \int \frac{\mathrm{d}^{3} \mathbf{p}_{a}}{2 E_{a}} \frac{\mathrm{d}^{3} \mathbf{p}_{b}}{2 E_{b}} \frac{\mathrm{d}^{3} \mathbf{p}_{c}}{2 E_{c}} \frac{\mathrm{d}^{3} \mathbf{p}_{\tilde{G}}}{2 E_{\tilde{G}}}|\mathcal{M}|^{2} f_{a} f_{b}\left(1 \pm f_{c}\right) \\
& \times \delta^{4}\left(P_{a}+P_{b}-P_{c}-P_{\tilde{G}}\right),
\end{aligned}
$$

where $f_{i}$ stands for the usual Bose and Fermi statistical densities,

$$
f_{B \mid F}=\frac{1}{e^{\frac{E}{T}} \mp 1} .
$$

In the temperature range of interest, all particles but the gravitino are in thermal equilibrium. For the gravitino, the statistical factor $f_{\tilde{G}}$ is negligible. Thus, $1-f_{\tilde{G}} \simeq 1$, as it is already used in (6). Furthermore, backward reactions are neglected. In addition, the simplification $1 \pm f_{c} \simeq 1$ is usually applied, making the analytic calculation of (6) possible. In our case, there is no such reason. We keep the factor $1 \pm f_{c}$ and consequently we proceed calculating the subtracted rate numerically [36].

The contribution of the processes $\mathrm{A}$ and $\mathrm{B}$, for each gauge group, can be read from Table I as

$\left|\mathcal{M}_{A, \mathrm{sub}}\right|^{2}+\left|\mathcal{M}_{B, \mathrm{sub}}\right|^{2}=\frac{g_{N}^{2}}{M_{\mathrm{P}}^{2}}\left(1+\frac{m_{\lambda_{N}}^{2}}{3 m_{3 / 2}^{2}}\right) C_{N}(-s+2 t)$.

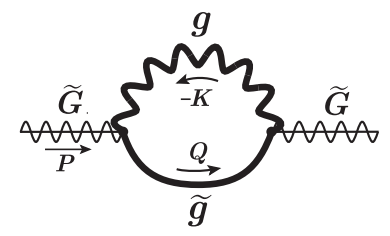

FIG. 1. The one-loop thermally corrected gravitino self-energy (D-graph) for the case of $S U(3)_{c}$. The thick gluon and gluino lines denote resummed thermal propagators. In our calculation, we have taken also into account the equivalent in $S U(2)_{L}$ and $U(1)_{Y}$.

In (8), a factor $1 / 2$ is already included for the process $A$ due to the two identical incoming particles. Substituting (8) in (6), the subtracted rate is obtained as

$\gamma_{\text {sub }}=\frac{T^{6}}{M_{\mathrm{P}}^{2}} \sum_{N=1}^{3} g_{N}^{2}\left(1+\frac{m_{\lambda_{N}}^{2}}{3 m_{3 / 2}^{2}}\right) C_{N}\left(-\mathcal{C}_{\mathrm{BBF}}^{s}+2 \mathcal{C}_{\mathrm{BFB}}^{t}\right)$.

The numerical factors, calculated by using the Cuba library [37], are $\mathcal{C}_{\mathrm{BBF}}^{s}=0.25957 \times 10^{-3}$ and $\mathcal{C}_{\mathrm{BFB}}^{t}=$ $-0.13286 \times 10^{-3}$. The subscripts $\mathrm{B}$ and $\mathrm{F}$ specify if the particles are bosons or fermions, respectively, and the superscripts determine if the squared amplitude is proportional to $s$ or $t$. It is easy to see that our result for the subtracted part unlike in [26] is negative. This is not unphysical, since the total rate and not the subtracted one is bound to be positive.

\section{B. The $D$-graph contribution}

As it has been discussed above, Eq. (3) describes the relation between the $D$-graph and the sum of the squared amplitudes for the $s, t$, and $u$ channels. In the $D$-graph contribution, we will implement the resummed thermal corrections to the gauge boson and gaugino propagators [38]. Although in Fig. 1 the gluino-gluon thermal loop is displayed, the contributions of all the gauge groups have been included in our analysis. The momentum flow used to calculate the $D$-graph can be depicted in Fig. 1. That is $\tilde{G}(P) \rightarrow g(K)+\tilde{g}(Q)$, with $P=(p, p, 0,0)$, $K=\left(k_{0}, k \cos \theta_{k}, k \sin \theta_{k}, 0\right)$ and $Q=\left(q_{0}, q \cos \theta_{q}, q \sin \theta_{q}, 0\right)$, where $\theta_{k, q}$ are the polar angles of the corresponding 3-momenta $\mathbf{k}, \mathbf{q}$ in spherical coordinates.

The non-time-ordered gravitino self-energy $\Pi^{<}(P)$ can be expressed in terms of the thermally resummed gaugino ${ }^{*} S^{<}(Q)$ and gauge boson ${ }^{*} D_{\mu \nu}^{<}(K)$ propagators as [21,26]

$$
\Pi^{<}(P)=\frac{1}{16 M_{P}^{2}} \sum_{N=1}^{3} n_{N}\left(1+\frac{m_{\lambda_{N}}^{2}}{3 m_{3 / 2}^{2}}\right) \int \frac{\mathrm{d}^{4} K}{(2 \pi)^{4}} \operatorname{Tr}\left[P\left[K, \gamma^{\mu}\right]^{*} S^{<}(Q)\left[K, \gamma^{\nu}\right]^{*} D_{\mu \nu}^{<}(K)\right]
$$

where 


$$
\begin{aligned}
& { }^{*} S^{<}(Q)=\frac{f_{F}\left(q_{0}\right)}{2}\left[\left(\gamma_{0}-\boldsymbol{\gamma} \cdot \mathbf{q} / q\right) \rho_{+}(Q)+\left(\gamma_{0}+\boldsymbol{\gamma} \cdot \mathbf{q} / q\right) \rho_{-}(Q)\right], \\
& { }^{*} D_{\mu \nu}^{<}(K)=f_{B}\left(k_{0}\right)\left[\Pi_{\mu \nu}^{T} \rho_{T}(K)+\Pi_{\mu \nu}^{L} \frac{k^{2}}{K^{2}} \rho_{L}(K)+\xi \frac{K_{\mu} K_{\nu}}{K^{4}}\right],
\end{aligned}
$$

with $\xi$ being the gauge parameter, taken $\xi=1$ [35] in our calculation and $n_{N}=\{1,3,8\} . \Pi_{\mu \nu}^{L}, \Pi_{\mu \nu}^{T}, \rho_{L, T}$, and $\rho_{ \pm}$are the longitudinal, the transverse projectors, and the spectral densities for the bosons and fermions, respectively. To compute the production rate related to the $D$-graph $\gamma_{D}$, we will use its definition [39]

$$
\gamma_{D}=\int \frac{\mathrm{d}^{3} \mathbf{p}}{2 p_{0}(2 \pi)^{3}} \Pi^{<}(p)
$$

and after appropriate manipulations [40], we obtain

$$
\begin{aligned}
\gamma_{\mathrm{D}}= & \frac{1}{4(2 \pi)^{5} M_{\mathrm{P}}^{2}} \sum_{N=1}^{3} n_{N}\left(1+\frac{m_{\lambda_{N}}^{2}}{3 m_{3 / 2}^{2}}\right) \int_{0}^{\infty} \mathrm{d} p \int_{-\infty}^{\infty} \mathrm{d} k_{0} \int_{0}^{\infty} \mathrm{d} k \int_{|k-p|}^{k+p} \mathrm{~d} q k f_{B}\left(k_{0}\right) f_{F}\left(q_{0}\right) \\
& \times\left[\rho_{L}(K) \rho_{-}(Q)(p-q)^{2}\left((p+q)^{2}-k^{2}\right)+\rho_{L}(K) \rho_{+}(Q)(p+q)^{2}\left(k^{2}-(p-q)^{2}\right)\right. \\
& +\rho_{T}(K) \rho_{-}(Q)\left(k^{2}-(p-q)^{2}\right)\left(\left(1+k_{0}^{2} / k^{2}\right)\left(k^{2}+(p+q)^{2}\right)-4 k_{0}(p+q)\right) \\
& \left.+\rho_{T}(K) \rho_{+}(Q)\left((p+q)^{2}-k^{2}\right)\left(\left(1+k_{0}^{2} / k^{2}\right)\left(k^{2}+(p-q)^{2}\right)-4 k_{0}(p-q)\right)\right],
\end{aligned}
$$

where $q_{0}=p-k_{0}$.

The spectral functions $\rho_{L, T}$ and $\rho_{ \pm}$can be found in Eq. (3.7) in [26]. The thermally corrected one-loop selfenergy for gauge bosons, scalars, and fermions that we have used in calculating these spectral functions can be found in [42-47]. Comparing (13) with the corresponding analytical result given in Eqs. (4.6) and (4.7) in [26], one can notice that they differ on the overall factor and on the number of independent phase-space integrations. Our analytical result has been checked using various frames for the momenta flow into the loop.

\section{The top Yukawa rate}

The production rate resulting from the top-quark Yukawa coupling $\lambda_{t}$ is given by [26]

$$
\gamma_{\text {top }}=\frac{T^{6}}{M_{\mathrm{P}}^{2}} 72 \mathcal{C}_{\mathrm{BBF}}^{s} \lambda_{t}^{2}\left(1+\frac{A_{t}^{2}}{3 m_{3 / 2}^{2}}\right)
$$

where $A_{t}$ is the trilinear stop supersymmetry breaking soft parameter and $\mathcal{C}_{\mathrm{BBF}}^{s}=0.25957 \times 10^{-3}$. Since this contribution stems from the process squark-squark $\rightarrow$ Higgsinogravitino, only the numerical factor $\mathcal{C}_{\mathrm{BBF}}^{s}$ is involved.

\section{THE PARAMETRIZATION OF THE RESULT}

Following [6], we parametrize the results (9) and (13) using the gauge couplings $g_{1}, g_{2}$, and $g_{3}$. Thus,

$$
\gamma_{\mathrm{sub}}+\gamma_{\mathrm{D}}=\frac{3 \zeta(3)}{16 \pi^{3}} \frac{T^{6}}{M_{\mathrm{P}}^{2}} \sum_{N=1}^{3} c_{N} g_{N}^{2}\left(1+\frac{m_{\lambda_{N}}^{2}}{3 m_{3 / 2}^{2}}\right) \ln \left(\frac{k_{N}}{g_{N}}\right),
$$

where the constants $c_{N}$ and $k_{N}$ depend on the gauge group and their values are given in Table II. In Fig. 2, we summarize our numerical results for the gravitino production rates divided by $Y_{N} T^{6} / M_{\mathrm{P}}{ }^{2}$. Especially, for the case of the top Yukawa contribution, in $Y_{N}$ the $m_{\lambda_{N}}^{2}$ has to be replaced by $A_{t}^{2}$. The colored solid curves represent the $S U(3)_{c}$ (red), $S U(2)_{L}$ (blue), and $U(1)_{Y}$ (green) rates given by (15) and the top Yukawa rate (purple) given by (14), while the black solid curve is the total result given by (5). The dashed black curve corresponds to the total result from [26]. For the sake of comparison, we have also chosen $\lambda_{t}=0.7$ [48].

Despite the analytical and numerical discrepancies with [26], it is interesting that our result for the total gravitino production rate is only $5 \%-11 \%$ smaller than that in [26]. Being unable to explain this quantitively in details, we assume that the aforementioned differences have opposing effects on the total result. For convenience, in Fig. 2, universal gauge coupling unification is assumed at the grand unification (GUT) scale $\simeq 2 \times 10^{16} \mathrm{GeV}$, but certainly the result in (15) can be used independently of this

TABLE II. The values of the constants $c_{N}$ and $k_{N}$ that parametrize our result (15) for the subtracted and the $D$-graph part. Each line corresponds to the particular gauge group, $U(1)_{Y}$, $S U(2)_{L}$, or $S U(3)_{c}$.

\begin{tabular}{lcc}
\hline \hline Gauge group & $c_{N}$ & $k_{N}$ \\
\hline$U(1)_{Y}$ & 41.937 & 0.824 \\
$S U(2)_{L}$ & 68.228 & 1.008 \\
$S U(3)_{c}$ & 21.067 & 6.878 \\
\hline \hline
\end{tabular}




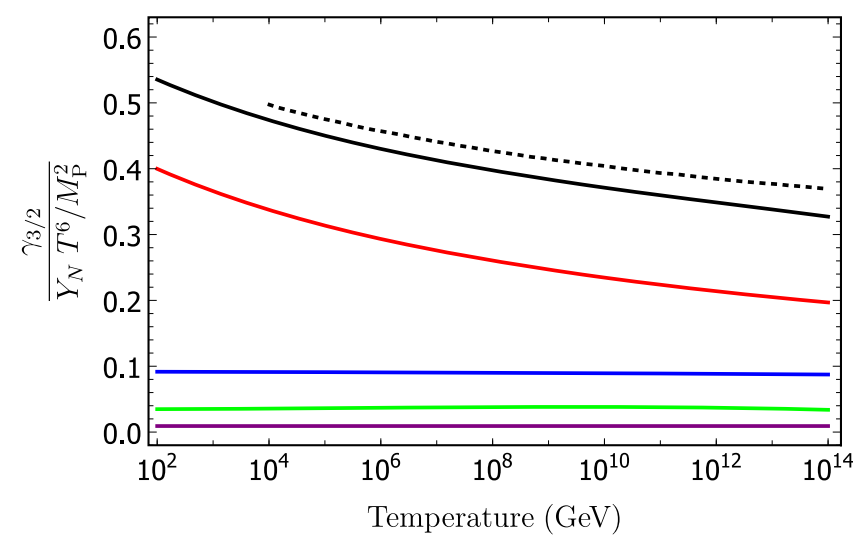

FIG. 2. The gravitino production rates divided by $Y_{N} T^{6} / M_{\mathrm{P}}{ }^{2}$. The solid curves represent in order the total rate (black) given by (5), the $S U(3)_{c}$ (red), $S U(2)_{L}$ (blue), and $U(1)_{Y}$ (green) rates given by (15), and the top Yukawa rate (purple) given by (14). The upper dashed curve is the total production rate obtained in [26]. The top Yukawa coupling $\lambda_{t}$ has been taken equal to 0.7 so that our result can be directly compared with that in [26].

assumption. Equation (15) along with the numbers in Table II is the main result of this paper.

\section{THE GRAVITINO ABUNDANCE}

The Boltzmann equation for the gravitino number density $n_{3 / 2}$ is

$$
\dot{n}_{3 / 2}+3 H n_{3 / 2}=\gamma_{3 / 2},
$$

where $H$ is the Hubble constant and the dot denotes time differentiation. The gravitino abundance is defined as

$$
Y_{3 / 2}=\frac{n_{3 / 2}}{n_{\mathrm{rad}}},
$$

with $n_{\text {rad }}=\zeta(3) T^{3} / \pi^{2}$. Substituting (17) into (16), we obtain that the gravitino abundance for $T \ll T_{\text {reh }}$ is given by [6]

$$
Y_{3 / 2}(T) \simeq \frac{\gamma_{3 / 2}\left(T_{\text {reh }}\right)}{H\left(T_{\text {reh }}\right) n_{\text {rad }}\left(T_{\text {reh }}\right)} \frac{g_{* S}(T)}{g_{* S}\left(T_{\text {reh }}\right)},
$$

where $g_{* s}$ are the effective entropy degrees of freedom in the minimal supersymmetric Standard Model (MSSM). In this, we assumed that inflaton decays are instantaneous, as the Universe is thermalizing. In [6,50], the case of not instantaneous inflaton decay has been taken into account. In particular, following [50], in the case of gravitino DM, a correction factor $\sim 10 \%$ is expected for not instantaneous inflaton decays.

Following the latest data from the Planck satellite, the cosmological accepted value for the DM density in the Universe is $\Omega_{\mathrm{DM}} h^{2}=0.1198 \pm 0.0012$ [51]. Assuming that the thermal gravitino abundance amounts to the observed DM, we obtain that

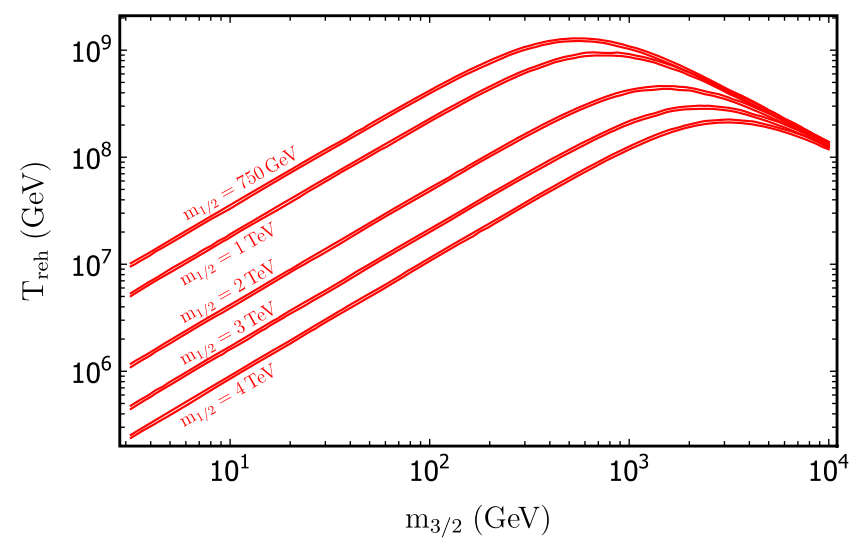

FIG. 3. The cosmologically accepted $3 \sigma$ regions for the gravitino thermal abundance for various values of $m_{1 / 2}$ between $750 \mathrm{GeV}$ and $4 \mathrm{TeV}$. The trilinear coupling $A_{t}$ has been ignored and the top Yukawa coupling is $\lambda_{t}=0.7$.

$$
\begin{aligned}
\Omega_{\mathrm{DM}} h^{2} & =\frac{\rho_{3 / 2}\left(t_{0}\right) h^{2}}{\rho_{\mathrm{cr}}}=\frac{m_{3 / 2} Y_{3 / 2}\left(T_{0}\right) n_{\mathrm{rad}}\left(T_{0}\right) h^{2}}{\rho_{\mathrm{cr}}} \\
& \simeq 1.33 \times 10^{24} \frac{m_{3 / 2} \gamma_{3 / 2}\left(T_{\mathrm{reh}}\right)}{T_{\text {reh }}^{5}},
\end{aligned}
$$

where $\rho_{\text {cr }}=3 H_{0}^{2} M_{\mathrm{P}}^{2}$ is the critical energy density, $H_{0}=$ $100 \mathrm{hkm} /(\mathrm{s} \mathrm{Mpc})$ is the Hubble constant, and $T_{0}=$ $2.725 \mathrm{~K}$ the cosmic microwave background temperature today. The entropy degrees of freedom at the associated temperatures are $g_{* s}\left(T_{0}\right)=43 / 11$ and $g_{* s}\left(T_{\text {reh }}\right)=915 / 4$. The last number equals to the effective energy degrees of freedom for $H\left(T_{\text {reh }}\right)$ in the MSSM too. Figure 3 illustrates the $3 \sigma$ regions resulting from (19) for various values of $m_{1 / 2}$. In this figure, the trilinear coupling $A_{t}$ has been ignored and the top Yukawa coupling is $\lambda_{t}=0.7$, as previously. As before, gauge coupling unification is assumed, as well as a universal gaugino mass $m_{1 / 2}$ at the GUT scale.

For large gravitino mass, the reheating temperature is $m_{1 / 2}$ independent, as the characteristic factor $m_{\lambda_{N}}^{2} /\left(3 m_{3 / 2}^{2}\right)$ becomes negligible for $m_{1 / 2} \ll m_{3 / 2}$. Assuming that $m_{1 / 2} \gtrsim 750 \mathrm{GeV}$, as it is suggested by the recent LHC data $[52,53]$ on gluino searches, from Fig. 3, we infer that for maximum $T_{\text {reh }} \simeq 10^{9} \mathrm{GeV}$ the corresponding gravitino mass is $m_{3 / 2} \simeq 550 \mathrm{GeV}$. Allowing for a reheating temperature an order of magnitude smaller, $T_{\text {reh }} \simeq 10^{8} \mathrm{GeV}$, for the same gravitino mass, $m_{1 / 2}$ can go up to $3-4 \mathrm{TeV}$.

\section{CONCLUSIONS}

In this paper, we have calculated the gravitino thermal abundance, using the full one-loop thermally corrected gravitino self-energy. Having rectified the main analytical formulas for the gravitino production rate, we have computed it numerically without approximation. We offer a simple and useful parametrization of our final result. In the 
context of minimal supergravity models, assuming gaugino mass unification, we have updated the bounds on the reheating temperature for certain gravitino masses. In particular, saturating the current LHC gluino mass limit $m_{\tilde{g}} \gtrsim 2100 \mathrm{GeV}$, we find that a maximum reheating temperature $T_{\text {reh }} \simeq 10^{9} \mathrm{GeV}$ is compatible to a gravitino mass $m_{3 / 2} \simeq 500-600 \mathrm{GeV}$.

It should be noted that trying to constrain the reheating temperature by applying the cosmological data on gravitino DM scenarios illuminates us whether thermal leptogenesis is a possible mechanism for generating baryon asymmetry or not. Successful thermal leptogenesis requires high temperature, $T_{\text {reh }} \gtrsim 2 \times 10^{9} \mathrm{GeV}$ [54-56], which is marginally bigger than the maximum reheating temperature obtained in our model using the lowest $m_{1 / 2}$ mass demonstrated in the recent LHC data [52,53]. In any case, there are many alternative models for baryogenesis. In addition, as it has been pointed out before, the thermal gravitino abundance is in general a part of the whole DM density and the inclusion of other components will affect the phenomenological analysis.

\section{ACKNOWLEDGMENTS}

The authors thank A. Lahanas and J. Pradler for useful discussions. This research is cofinanced by Greece and the European Union (European Social Fund - ESF) through the Operational Programme "Human Resources Development, Education and Lifelong Learning" in the context of the project "Strengthening Human Resources Research Potential via Doctorate Research-2nd Cycle" (MIS-5000432), implemented by the State Scholarships Foundation (IKY). This research work was supported by the Hellenic Foundation for Research and Innovation (H.F.R.I.) under the "First Call for H.F.R.I. Research Projects to support Faculty members and Researchers and the procurement of high-cost research equipment grant" (Project No. 824).
[1] R. Kallosh, L. Kofman, A. D. Linde, and A. Van Proeyen, Gravitino production after inflation, Phys. Rev. D 61, 103503 (2000).

[2] G. F. Giudice, A. Riotto, and I. Tkachev, Thermal and nonthermal production of gravitinos in the early universe, J. High Energy Phys. 11 (1999) 036.

[3] H. P. Nilles, M. Peloso, and L. Sorbo, Nonthermal Production of Gravitinos and Inflatinos, Phys. Rev. Lett. 87, 051302 (2001).

[4] M. Kawasaki, F. Takahashi, and T. T. Yanagida, Gravitino overproduction in inflaton decay, Phys. Lett. B 638, 8 (2006).

[5] M. Endo, M. Kawasaki, F. Takahashi, and T. T. Yanagida, Inflaton decay through supergravity effects, Phys. Lett. B 642, 518 (2006).

[6] J. Ellis, M. A. G. Garcia, D. V. Nanopoulos, K. A. Olive, and M. Peloso, Post-inflationary gravitino production revisited, J. Cosmol. Astropart. Phys. 03 (2016) 008.

[7] E. Dudas, Y. Mambrini, and K. Olive, Case for an EeV Gravitino, Phys. Rev. Lett. 119, 051801 (2017).

[8] K. Kaneta, Y. Mambrini, and K. A. Olive, Radiative production of nonthermal dark matter, Phys. Rev. D 99, 063508 (2019).

[9] M. Kawasaki, K. Kohri, T. Moroi, and A. Yotsuyanagi, Big-bang nucleosynthesis and gravitino, Phys. Rev. D 78, 065011 (2008).

[10] M. Kawasaki, K. Kohri, T. Moroi, and Y. Takaesu, Revisiting big-bang nucleosynthesis constraints on long-lived decaying particles, Phys. Rev. D 97, 023502 (2018).

[11] R. H. Cyburt, J. R. Ellis, B. D. Fields, K. A. Olive, and V. C. Spanos, Bound-state effects on light-element abundances in gravitino dark matter scenarios, J. Cosmol. Astropart. Phys. 11 (2006) 014.
[12] R. H. Cyburt, J. Ellis, B. D. Fields, F. Luo, K. A. Olive, and V.C. Spanos, Metastable charged sparticles and the cosmological ${ }^{7} \mathrm{Li}$ problem, J. Cosmol. Astropart. Phys. 12 (2012) 037.

[13] S. Weinberg, Cosmological Constraints on the Scale of Supersymmetry Breaking, Phys. Rev. Lett. 48, 1303 (1982).

[14] J. R. Ellis, J. E. Kim, and D. V. Nanopoulos, Cosmological gravitino regeneration and decay, Phys. Lett. 145B, 181 (1984).

[15] M. Y. Khlopov and A. D. Linde, Is it easy to save the gravitino?, Phys. Lett. 138B, 265 (1984).

[16] T. Moroi, H. Murayama, and M. Yamaguchi, Cosmological constraints on the light stable gravitino, Phys. Lett. B 303, 289 (1993).

[17] M. Kawasaki and T. Moroi, Gravitino production in the inflationary universe and the effects on big bang nucleosynthesis, Prog. Theor. Phys. 93, 879 (1995).

[18] T. Moroi, Effects of the gravitino on the inflationary universe, Ph.D. thesis, Tohoku University, 1995.

[19] J. R. Ellis, D. V. Nanopoulos, K. A. Olive, and S. J. Rey, On the thermal regeneration rate for light gravitinos in the early universe, Astropart. Phys. 4, 371 (1996).

[20] M. Bolz, W. Buchmuller, and M. Plumacher, Baryon asymmetry and dark matter, Phys. Lett. B 443, 209 (1998).

[21] M. Bolz, A. Brandenburg, and W. Buchmuller, Thermal production of gravitinos, Nucl. Phys. B606, 518 (2001); Erratum, Nucl. Phys. B790, 336 (2008).

[22] M. Bolz, Thermal production of gravitinos, Ph.D. thesis, Hamburg University, 2000.

[23] F. D. Steffen, Gravitino dark matter and cosmological constraints, J. Cosmol. Astropart. Phys. 09 (2006) 001. 
[24] J. Pradler and F. D. Steffen, Thermal gravitino production and collider tests of leptogenesis, Phys. Rev. D 75, 023509 (2007).

[25] J. Pradler and F. D. Steffen, Constraints on the reheating temperature in gravitino dark matter scenarios, Phys. Lett. B 648, 224 (2007).

[26] V.S. Rychkov and A. Strumia, Thermal production of gravitinos, Phys. Rev. D 75, 075011 (2007).

[27] J. Pradler, Electroweak contributions to thermal gravitino production, Diploma thesis, University of Vienna, 2006.

[28] G. F. Giudice and R. Rattazzi, Theories with gauge mediated supersymmetry breaking, Phys. Rep. 322, 419 (1999).

[29] K. Choi, K. Hwang, H. B. Kim, and T. Lee, Cosmological gravitino production in gauge mediated supersymmetry breaking models, Phys. Lett. B 467, 211 (1999).

[30] T. Asaka, K. Hamaguchi, and K. Suzuki, Cosmological gravitino problem in gauge mediated supersymmetry breaking models, Phys. Lett. B 490, 136 (2000).

[31] K. Jedamzik, M. Lemoine, and G. Moultaka, Gravitino dark matter in gauge mediated supersymmetry breaking, Phys. Rev. D 73, 043514 (2006).

[32] E. Braaten and R. D. Pisarski, Soft amplitudes in hot gauge theories: A general analysis, Nucl. Phys. B337, 569 (1990).

[33] E. Braaten and T. C. Yuan, Calculation of Screening in a Hot Plasma, Phys. Rev. Lett. 66, 2183 (1991).

[34] T. Lee and G. H. Wu, Interactions of a single Goldstino, Phys. Lett. B 447, 83 (1999).

[35] As it has been pointed out in [26] the splitting of the amplitudes in resummed and non-resummed contributions violates the gauge invariance. Therefore a gauge dependence of the result is expected.

[36] As it was argued in [22] and we have checked numerically, the effect of taking into account the statistical factor $f_{c}$ can be around $-10 \%(+20 \%)$ if $c$ is a fermion (boson).

[37] T. Hahn, CUBA: A library for multidimensional numerical integration, Comput. Phys. Commun. 168, 78 (2005).

[38] Like in [26] using the gravitino polarization sum (1), we nullify the corresponding quark-squark $D$-graph.

[39] M. L. Bellac, Thermal Field Theory, Cambridge Monographs on Mathematical Physics (Cambridge University Press, Cambridge, United Kingdom, 2011).

[40] In principle, we use the $\delta$-functions to reduce the number of the phase-space integrations. Further details will appear in [41].

[41] H. Eberl, I. D. Gialamas, and V. C. Spanos (to be published).

[42] H. A. Weldon, Covariant calculations at finite temperature: The relativistic plasma, Phys. Rev. D 26, 1394 (1982).
[43] H. A. Weldon, Effective fermion masses of order $g T$ in high temperature gauge theories with exact chiral invariance, Phys. Rev. D 26, 2789 (1982).

[44] H. A. Weldon, Dynamical holes in the quark-gluon plasma, Phys. Rev. D 40, 2410 (1989).

[45] H. A. Weldon, Structure of the gluon propagator at finite temperature, Ann. Phys. (N.Y.) 271, 141 (1999).

[46] A. Peshier, K. Schertler, and M. H. Thoma, One loop selfenergies at finite temperature, Ann. Phys. (N.Y.) 266, 162 (1998).

[47] H. A. Weldon, Structure of the quark propagator at high temperatures, Phys. Rev. D 61, 036003 (2000).

[48] The gauge framework of our calculation is the minimal supersymmetric Standard Model gauge group $S U(3)_{c} \times$ $S U(2)_{L} \times U(1)_{Y}$. Using a different gauge structure like $S U(5)$ or flipped $S U(5)$ [49] apparently constitutes a completely new calculation.

[49] J. Ellis, M. A. G. Garcia, N. Nagata, D. V. Nanopoulos, and K. A. Olive, Superstring-inspired particle cosmology: inflation, neutrino masses, leptogenesis, dark matter \& the SUSY scale, J. Cosmol. Astropart. Phys. 01 (2020) 035.

[50] M. A. G. Garcia, Y. Mambrini, K. A. Olive, and M. Peloso, Enhancement of the dark matter abundance before reheating: Applications to gravitino dark matter, Phys. Rev. D 96, 103510 (2017).

[51] N. Aghanim et al. (Planck Collaboration), Planck 2018 results. VI. Cosmological parameters, Astron. Astrophys. 641, A6 (2020).

[52] M. Aaboud et al. (ATLAS Collaboration), Search for supersymmetry in final states with missing transverse momentum and multiple $b$-jets in proton-proton collisions at $\sqrt{s}=13 \mathrm{TeV}$ with the ATLAS detector, J. High Energy Phys. 06 (2018) 107.

[53] A. M. Sirunyan et al. (CMS Collaboration), Search for supersymmetry in proton-proton collisions at $13 \mathrm{TeV}$ in final states with jets and missing transverse momentum, J. High Energy Phys. 10 (2019) 244.

[54] G. F. Giudice, A. Notari, M. Raidal, A. Riotto, and A. Strumia, Towards a complete theory of thermal leptogenesis in the SM and MSSM, Nucl. Phys. B685, 89 (2004).

[55] W. Buchmuller, R. D. Peccei, and T. Yanagida, Leptogenesis as the origin of matter, Annu. Rev. Nucl. Part. Sci. 55, 311 (2005).

[56] S. Antusch and A. M. Teixeira, Towards constraints on the SUSY seesaw from flavour-dependent leptogenesis, J. Cosmol. Astropart. Phys. 02 (2007) 024. 